\title{
Optimization Design Method of Noise Control on Fine-Pitch Gear
}

\author{
Tie Wang ${ }^{*}$, Fu Qiang Zhao, Ruiliang Zhang and Lili Qi \\ Gear Research Institute, Taiyuan University of Technology, Taiyuan 030024, China
}

\begin{abstract}
Proceed from reducing the noise of automotive gears, a new method of the fine-pitch tooth profile optimization is proposed. Under the premise of the constant gear parameter values of the transmission ratio, modulus, and pressure angle, the method considers the minimum noise objective value as the design object, and the constraint conditions are established such as the noninterference in meshing, the non-undercutting, and the non-pointed in the tip and so on. Then the coefficients of addendum and modification are optimized with the purpose of increasing the contact ratio value and full-depth tooth. And it is necessary for this paper to conduct a strength analysis on the design result. The analysis result shows that the new method can not only reduce the noise, also ensure the high quality of the automotive gear strength.
\end{abstract}

Keywords: Automotive gear, noise, tooth profile parameter, fuzzy optimization.

\section{INTRODUCTION}

At present it has become one of the critically needed problems of the international automotive gear research to design the high-strength and low-noise gear. Fine-pitch gears have a small modulus, small pressure angle, and a big fulldepth tooth [1], which cause the contact ratio of the transverse increased so as to improve the gear strength and reduce the noise. Fine-pitch tooth gear has a wide application prospect in automobile transmission industry. Xianbo Zhengzhuang made a detailed presentation on the characteristics and experiments of the gear of this kind [2]. Eaton has realized the production of fine-pitch tooth gear transmission. As there are many design dimensions that impact the noise and strength of the fine-pitch gear, and especially on heavy-duty automotive transmission there are various requirements for gear design. So the reasonable parameter value is one of the main directions of research on fine-pitch tooth profile.

There are a number of factors influencing gear noise, such as manufacturing accuracy, resonance and bearing accuracy etc. It should be considered in gear design. The noise can be effectively reduced by using reasonable modulus and proper contact ratio, or modifying tooth profile. Meshing noise is an inherent characteristic of gear. Through the adjustment of related parameter in the design, it appears feasible to achieve the purpose of reducing the noise, by the way of decreasing and absorbing the vibration. Based on the traditional design, conventional reliability design and optimization theory, the fuzzy reliability optimal design introduces the fuzzy theory and forms a modern design method [3]. The mathematical model of this method can be able to reasonably reflect the influence of the fuzzy factors. Generally the gear design is influenced by the design level, manufacture, working conditions and so on [4]. As these factors is uncertain in

*Address correspondence to this author at the Gear Research Institute, Taiyuan University of Technology, Taiyuan 030024, China; Tel: +86-3516010580(Office), +86-13934530090(Mobile); Fax: +86-351-6010580; E-mail: wangtie57@163.com boundaries, the fuzzy reliability optimization design considers the main factors which affect the design, carry on the abstraction of the mathematical model according to the design target, and can offer reasonable and practical reference value.

\section{TOOTH PROFILE PARAMETERS INFLUENCE ON MESHING NOISE}

\subsection{Pressure Angle and Modulus}

According to the gear standards in different countries, the pressure angle which is about 20 is generally considered. If pressure angle is reduced, the gear stiffness is decreased and dynamic load is similarly, which is produced by the meshing of gears. In addition, taking machining accuracy into consideration contact ratio of gear and cutter has closely relationship with the gear accuracy obtained, that is to say, the greater contact ratio, the less tooth profile error is. So the pressure angle smaller than 20 is used in the transmission of trapezoidal gear. Modulus is one of the basic parameters in the calculation, which reflects the size of pitch. After giving reasonable gear numbers, small modulus is better than big for tooth profile to be finer and thinner. What is more, the small modulus can not only increase contact ratio of the transverse, also enhance gear flexibility through making tooth root thinner. So it is easily to absorb the gear vibration.

\subsection{Addendum Coefficient}

On the basis of constant values of pressure angle and modulus, it is an important way to enlarge the contract ratio and thin the tooth profile to increase the addendum coefficient. In the standard gear design, the addendum coefficient usually adopts the compromise value 1 . But according to the design requirement, it is necessary to change the addendum coefficient to acquire the excellent performance. For the nonmodified gear with constant modulus, the addendum circle diameter and contact ratio can be increased by the way of enlarging addendum coefficient. 


\subsection{Modification Coefficient}

The changes of the modification coefficient can also realize the increase of the addendum circle diameter. For the gear with the same modulus and teeth number, the increasing modification coefficient with positive modification value will increase the addendum and the tip diameter, and also decrease the dedendum and root diameter so as to make the tooth profile thinner and higher. In addition, the positive modification value increases the tooth profile curvature radius, which can improve the tooth contact strength.

\subsection{Contract Ratio}

Niemann and Unterberger make a research on the straight gear noise [2]. The results shows that the greater contact ratio is, the lesser the dynamic load is, with lower meshing noise and higher strength. In addition, the larger of the contact ratio, the lower of the noise. When the contact ratio increases to 2 , the noise series decreases sharply, which is also called noise-elimination-gear. When the contact ratio is more than 2.2, the noise series will increase again. In summary, the contact ratio range of the fine-pitch gear is 2 to 2.2 .

\section{FINE-PITCH GEAR STRENGTH ANALYSIS}

\subsection{Tooth Surface Strength}

Generally speaking, with the contact ratio less than 2 in the high-precision gear pair, the maximum pinion tooth surface stress value is at the point of maximum load. With the contact ratio decreasing, the meshing point is gradually closed to the pinion interference point. So, theoretically, the allowable load of the tooth surface is decreased with the reduce of contact ratio due to the maximum stress increasing of the tooth surface. The contact ratio has an effect, which is primarily illustrated through experimental results, on the tooth surface strength. Niemann and Retting has also released the corresponding results which is shown in Fig. (1). The Fig. (1A), which is different from the Fig. (1B and 1C), shows the experimental result of the roller gear (DIN8-
9). The allowable load of the high gear is $60 \%$ higher than the standard gear with $20^{\circ}$ pressure angle, and only $10 \%$ to $15 \%$ lower than the standard gear with $28^{\circ}$ pressure angle.

Because when $\varepsilon$ is more than 2, the dynamic load is lesser, so this kind of high gears have a large allowable load. For $28^{\circ}$ standard high gear, it also has a large allowable load because of the big stiffness. Niemann uses quenched and grinding gear (DIN6) as shown in Fig. (1B). The Experiment results of quenched and grinding bevel gear (DIN6) is shown in Fig. (1C) when $\beta$ is $25^{\circ}$.

In Fig. (1), $\mathrm{H}$ stands for the high gear which $\varepsilon$ is 2.16 and $\mathrm{N}$ stands for the standard gear.

\subsection{Root Strength}

The root transition curved line is one of the main factors influenced bending strength. It is known that the full radius hob is more effective than two-corner hob on increasing tooth bending strength. In general, the greater the transition fillet is, the higher bending strength is. Taking machining efficiency into consideration, transition curved line, which is manufactured by full radius hob on tooth tip, is chosen on fine-pitch gear. In addition, the root bending strength is increased when the contact ratio is increased. The reason is that when the contact ratio is increased, the maximum load point of the outside which is also the outer point of single tooth meshing is far away from the tip. So the tooth profile coefficient is increased.

\section{FUZZY OPTIMUM DESIGN}

The current gear optimization design is usually on the basis of gear strength, and it cannot effectively reduce the meshing noise. For example, the optimal design takes the smallest volume as the target so as to acquire the minim mass, lowest cost, and highest reliability [4-7]. While the fine-pitch tooth profile fuzzy optimum designs is based on gear meshing theory and takes the reduction of noise as the goal. With the constant transmission ratio and normal center distance, this method increases the addendum so as to obtain

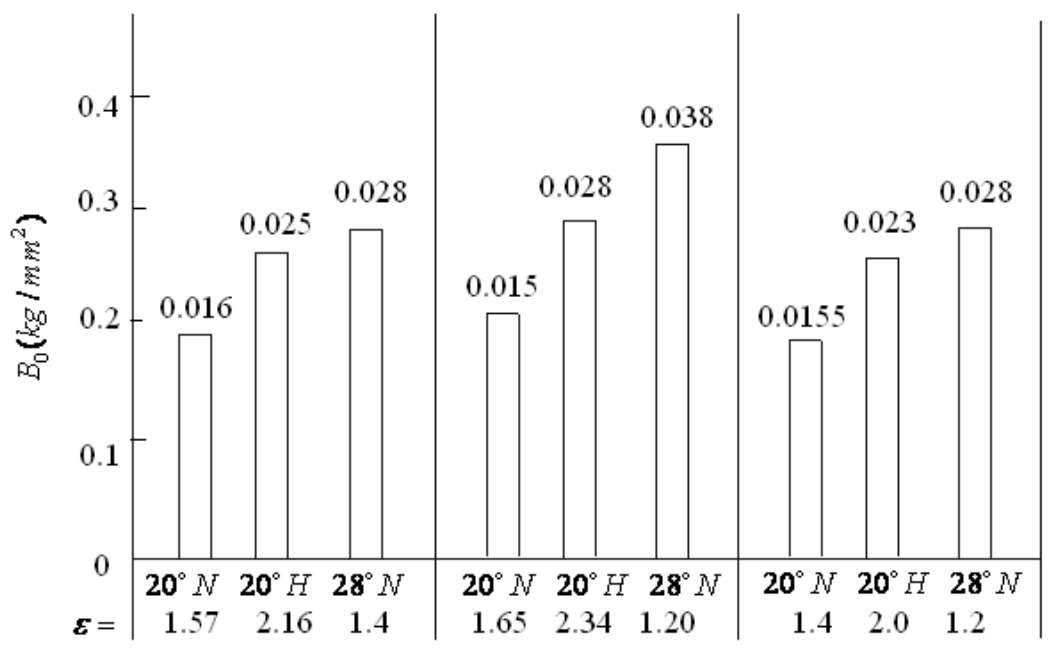

(A)

(B)

(C)

Fig. (1). Contact ratio influence on surface strength (2). 
higher gear strength and lower noise. What's more, the method takes into account the discrete and fuzzy of the main factors and constraints which affect the design in order to acquire a better synthetically result.

\subsection{Definition of Objective Function}

It is assumed that the objective function $F(X)$ is realvalued function in the domain $X$, which takes into account the slide of tooth ratio affected meshing noise and dependent the mutation of friction torque comprehensively. The slide of tooth ratio is one of the important investigating noise index. The tooth profile does not participate near the base circle of the pinion due to large slide ratio. When slide ratio of the pinion is similar to the gear wheel, the relative slide is hardly happened. On the other hand, the mutation of friction torque and noise are both maximum at the moment when tooth contact moving from the addendum profile to the dedendum profile. So it is advisable to reduce meshing noise through the way of the design of the addendum profile and dedendum profile. The objective function is,

$$
\begin{aligned}
F(X)= & \frac{d_{b 1}+r_{w b_{-} a \min }}{\sqrt{d_{b 1}^{2}+\left(2 a \sin \alpha_{t}-\sqrt{d_{a 2}^{2}-d_{b 2}^{2}}\right)^{2}}} \\
& +\frac{d_{b 2}+r_{w b_{-} a \min }}{\sqrt{d_{b 2}^{2}+\left(2 a \sin \alpha_{t}-\sqrt{d_{a 1}^{2}-d_{b 1}^{2}}\right)^{2}}} \\
& +\frac{2 \rho_{2 \max }-d_{b 2} \tan \alpha_{t}}{2 \rho_{1 \max }-d_{b 1} \tan \alpha_{t}}
\end{aligned}
$$

Where $\alpha_{t}$ is the meshing angle of the transverse, $\rho_{1 \max }$ is driving gear addendum circle tooth profile radius of curvature, and $\rho_{2 \max }$ is driven gear addendum circle tooth profile radius of curvature. And $r_{w b \_a m i n}$ is the minimum distance between meshing initial circle and base circle. Superscript 1 and 2 respectively stand for driving gear and driven gear.

The modification coefficient is usually limited by the contact ratio, the transition curve non-undercutting in the gear tip, the tip thickness and so on. In addition, the modification coefficient closed graph is used which connects working pressure angle, theoretical contact ratio and the modification coefficient. When gear number is given, according to limiting condition, the relation of modification coefficient is got. When meshing angel is given, $x_{1}$ and $x_{2}$ are linear, which is indicated by slashes, here,

$x_{1}=-x_{2}+F\left(\alpha_{w t}, \alpha\right)$

Where $F\left(\alpha_{w t}, \alpha\right)$ is a quadratic curve about $x_{1}$ and $x_{2}$.

Usually, the step is setting up the value range of the tooth number, the normal center distance, and the optimize parameters. Then the maximum of the objective function can be obtained, which is corresponded to the optimal parameters. Usually, the automobile gear modulus and pressure angle have corresponding standards. So it is not necessary to take them as design variables. Since the choice of design variables has a significantly influence on the result, this paper chooses the tooth profile parameters as design variables, which have a significant impact on the contact ratio and can be controlled easily, $x_{1}$ and $x_{2}$ are modification coefficients, $h_{\mathrm{a} 1}{ }^{*}$ and $h_{\mathrm{a} 2}{ }^{*}$ are tooth roof coefficients, here is,

$$
X=\left[x_{1}, x_{2}, x_{3}, x_{4}\right]^{T}=\left[x_{1}, x_{2}, h_{a 1}{ }^{*}, h_{a 2}{ }^{*}\right]^{T}
$$

According to experience and calculation, when the objective function values in the range of $(2,3)$, it is easily to control noise series in a better level.

\subsection{Definition of the Constraint Condition}

According to the factors that impact the contact ratio of the fine-pitch tooth, the constraints are established as follows:

(1) Boundary constraints for modification coefficient. It is,

$\underline{x_{i}} \leq x_{i} \leq \bar{x}_{i} \quad(i=1,2)$

(2) Boundary constraints for addendum coefficient. It is,

$1.2<h_{a j}{ }^{*}<1.5 \quad(j=1,2)$

(3) Boundary constraints for objective function value. It is,

$2<\varepsilon<2.2$

(4) The Constraints for the tip thickness.

This paper takes the $0.3 \mathrm{~m}$ tip thickness as the limited value of the maximum diameter, which is $s_{\text {amin }}=0.3 \mathrm{~m}$. The maximum diameter can be obtained, and normal tip thickness is

$$
g_{1,2}(X)=d_{a 1,2}\left(\frac{\pi+4 x_{1,2} \tan \alpha}{2 z_{1,2}}+i n v \alpha-i n v \alpha_{a 1,2}\right)-s_{a \text { min }} \geq \tilde{0}
$$

(5) The Constraints for the non-interference.

The additional constraints are as follows, to avoid interference caused by a narrow space width.

$$
g_{3,4}(X)=\tan \alpha_{w}-\tan \alpha-\frac{z_{2}}{z_{1}}\left(\tan \alpha_{a 2,1}-\tan \alpha_{w}\right)+\frac{4\left(h_{a}^{*}-x_{1,2}\right)}{z_{1,2} \sin 2 \alpha} \geq \tilde{0}
$$

(6) The Constraints for the non-undercut while cutting gear.

The minimum modification coefficients are,

$g_{5,6}(X)=x_{1,2}-x_{\min } \geq \tilde{0}$

\subsection{Mathematical Model}

Based on the above design variables, objective function and constraints, non-symmetric mathematical model is,

Find,

$$
X=\left[x_{1}, x_{2}, x_{3}, x_{4}\right]^{T}
$$

$\operatorname{Max} F(X)$

$$
\text { Subject to } \quad \underline{x_{i}} \leq x_{i} \leq \bar{x}_{i} \quad(i=1,2,3)
$$

$$
g_{i}(x) \geq 0 \quad(i=4,5,6,7,8,9)
$$




\subsection{Definition of Fuzzy Constraint Function}

The bound variables of the fine-pitch tooth are based on the experience and relevant references. So according to the fuzzy design theory, it is available to rationally establish the function that reflect the elements from not attached to attached, here

$\mu(x)= \begin{cases}0 & x \leq \underline{x_{l}} \\ \frac{x-\underline{x_{l}}}{\underline{x_{u}}-\underline{x_{l}}} & \underline{x_{l}}<x<\underline{x_{u}} \\ 1 & \underline{x_{u}}<x<\overline{x_{l}} \\ \overline{x_{u}}-x & -\overline{\bar{x}}<x<\overline{x_{u}} \\ \overline{x_{u}}-\overline{x_{l}} & x_{l}<\overline{x_{u}} \\ 0 & x<1\end{cases}$

It also could be amended according to the actual. The membership function that expresses the scope of the border is shown in Fig. (2). It is based on amplification coefficient method to determine the tolerance. The upper amplification coefficient is $\bar{\beta}=1.10$, and the lower amplification coefficient is $\underline{\beta}=0.8$. Then it will determine the upper and lower limits $\underline{x}_{l}$ and $\underline{x}_{u}$ of the upper boundary as well as the upper and lower limits $\overline{x_{l}}$ and $\overline{x_{u}}$ of the lower boundary.

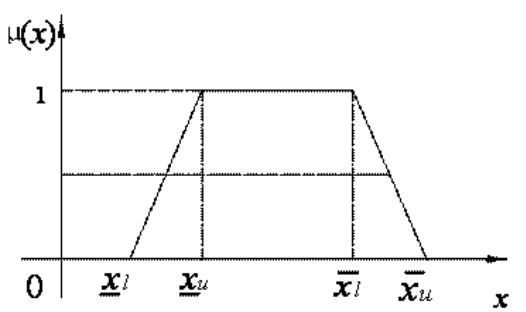

Fig. (2). Intermediate membership function.

\subsection{The Solution of the Fuzzy Optimization Design}

Design standards, manufacturing standards and material properties are the major sources of fuzziness of the fine-pitch tooth profile. As the design goals and constraints are nonsymmetric, this paper applies the optimizing level-cut-set method and the Matlab soft. In the fuzzy interval $G$, the membership degree $\mu_{\mathrm{G}}(g) \geqq \lambda$ constitutes the level set of $\lambda$ in the domain of real number. So there is a $\lambda^{*}$ as the optimization solution to make the design safe and reliable. Thus the paper changes the asymmetry fuzzy optimization into the optimizing level-cut-set in the process. And fine-pitch gear uses two-level fuzzy comprehensive evaluation. Here,

$G_{\lambda}=\left\{g \mid \mu_{G}(g) \geq \lambda, \lambda \in[0,1]\right\}$

(1) The 1st Fuzzy Comprehensive Evaluation. The factors, factor levels and their membership degree that affect the optimal level set $\lambda^{*}$ are shown in Table $\mathbf{1}$.

Sampling interval is adopted equal-step-discrete $h=0.05$. And the standby selected aggregate is,

$$
\lambda=\{0.1,0.2,0.3,0.4,0.5,0.6,0.7,0.8,0.9,1\}
$$

The factorial power sets are,

$A_{1}=\{0.15,0.5,0.35,0,0\}, A_{2}=\{0,0.35,0.5,0.15,0\}$,

$A_{3}=\{0.2,0.45,0.35,0,0\}, A_{4}=\{0.2,0.5,0.3,0,0\}$,

$A_{5}=\{0,0,0.25,0.5,0.25\}$.

Then, the grade evaluation matrix of each level factor is established.

Through the comprehensive judgment to the different grades of fuzzy subset of the $i^{\text {th }}$ factor, the 1 st fuzzy comprehensive evaluation sets can be obtained. That is,

$B_{i}=A_{i} \bullet R_{i}$

(2) The 2nd Fuzzy Comprehensive Evaluation. According to $B_{\mathrm{i}}$, the 2 nd fuzzy transformation matrix $R$ can be established.

The 2nd evaluation set $B$ can be obtained through calculating the impact factors, here

$$
\begin{aligned}
& B=A \bullet R=\left\{b_{j} \mid j=1,2, \cdots, 10\right\}
\end{aligned}
$$

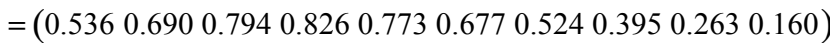

This paper makes a weighted average on the $\underline{\lambda}_{j}(j=1,2, \ldots$, $10)$, and compare the $\lambda^{*}$ with the standby selected aggregate. Thus the optimal level-cut set can be acquired. Thus the fuzzy optimization can be changed into the general optimal solution, here

$$
\lambda^{*}=\frac{\sum_{j=1}^{10} b_{j} \lambda_{j}}{\sum_{j=1}^{10} b_{j}}=0.392
$$

Table 1. Factors, Factor Levels and Membership Degree

\begin{tabular}{|c|c|c|c|c|c|c|c|c|c|c|}
\hline \multirow{2}{*}{ Factors } & \multicolumn{4}{|c|}{ Factor Levels } & \multicolumn{5}{c|}{ Membership Degree } \\
\cline { 2 - 13 } & $\mathbf{1}$ & $\mathbf{2}$ & $\mathbf{3}$ & $\mathbf{4}$ & $\mathbf{5}$ & $\mathbf{1}$ & $\mathbf{2}$ & $\mathbf{3}$ & $\mathbf{4}$ & $\mathbf{5}$ \\
\hline \hline Design & $\mathrm{B}$ & $\mathrm{G}$ & $\mathrm{N}$ & $\mathrm{P}$ & $\mathrm{W}$ & 0.25 & 0.5 & 0.35 & 0 & 0 \\
\hline Manufacture & $\mathrm{B}$ & $\mathrm{G}$ & $\mathrm{N}$ & $\mathrm{P}$ & $\mathrm{W}$ & 0.0 & 0.35 & 0.5 & 0.15 & 0 \\
\hline Material & $\mathrm{B}$ & $\mathrm{G}$ & $\mathrm{N}$ & $\mathrm{P}$ & $\mathrm{W}$ & 0.2 & 0.45 & 0.35 & 0 & 0 \\
\hline Importance & $\mathrm{B}$ & $\mathrm{G}$ & $\mathrm{N}$ & $\mathrm{P}$ & $\mathrm{W}$ & 0.2 & 0.5 & 0.3 & 0 & 0 \\
\hline Use condition & $\mathrm{B}$ & $\mathrm{G}$ & $\mathrm{N}$ & $\mathrm{P}$ & $\mathrm{W}$ & 0 & 0 & 0.3 & 0.5 & 0.2 \\
\hline
\end{tabular}

Factor levels: $\mathrm{B}$ is Better, $\mathrm{G}$ is good, $\mathrm{N}$ is normal, $\mathrm{P}$ is poor, $\mathrm{W}$ is worse. 
Table 2. Gear Parameters

\begin{tabular}{|c|c|c|c|c|c|}
\hline \multirow{2}{*}{ Project } & \multirow{2}{*}{ Symbol } & \multicolumn{2}{|c|}{ Original Design Gears } & \multicolumn{2}{|c|}{ Improved Design Gears } \\
\hline & & Driving & Driven & Driving & Driven \\
\hline Tooth Number & $\mathrm{z}$ & 29 & 37 & 29 & 37 \\
\hline Pressure Angle $\left[{ }^{\circ}\right]$ & $\alpha$ & \multicolumn{2}{|c|}{16} & \multicolumn{2}{|c|}{16} \\
\hline Helix Angle $\left[{ }^{\circ}\right]$ & $\beta$ & \multicolumn{2}{|c|}{30} & \multicolumn{2}{|c|}{30} \\
\hline Addendum Coef. & $h_{a}$ & 1.2 & 1.2 & 1.45 & 1.55 \\
\hline Tip Diameter [mm] & $d_{a}$ & 126.195 & 178.805 & 130.258 & 160.497 \\
\hline Tip Thickness [mm] & $S_{a}$ & 1.371 & 1.539 & 1.174 & 1.347 \\
\hline Objective Function & $F(x)$ & 2.552 & 2.436 & 1.882 & 1.876 \\
\hline
\end{tabular}

\section{NUMERICAL EXAMPLES}

This paper takes the 2nd hardened gear of TYXG-5T transmission as the research object. Center distance is $88 \mathrm{~mm}$, and pressure angel is 20 , then spiral angel is 24 . The original gear pair are a single tooth meshing. The fuzzy optimization mathematical model is established. After the model is transformed into the general optimization problem, this paper applies the Matlab software and orthogonal grid method to solve. In order to make operation easy, setting up two-tip coefficients changing with the same step. After gained improving design, the finite element method is adopted to solve gear stress value. Detailed data is shown in Table 2.

In comparisons with the optimization tooth parameters in Table 2, it is shown that the addendum is increased, the tooth profile becomes higher and thinner, and the theoretical meshing line turns longer. It also can be calculated that the contact ratio increases from 1.873 to 2.165 .

The bending stress value of original driving gear design is close to the one of driven gear design. After adjusting modification coefficient, the driving gear stress is decreased. In contrast, driven gear stress is increased. What is more, it can be seen from Table 2 that if modification coefficient of driven gear is 0 , the function value does not increase obviously with the purpose of reducing the mutation of friction torque influence on the noise.

\section{CONCLUSIONS}

(1) This paper applies the fuzzy reliability optimization design method to fine-pitch tooth profile optimization design. The method takes the lowest meshing noise as the design goals and the main geometric parameters as the optimized variables. Based on this, the paper sets up the asymmetry fuzzy optimization mathematical model of automotive tooth profile. The contact ratio and full depth tooth increase reasonably.

(2) After considering various affecting factors in the gear design, it is conclude that the optimal level-cut-set method can be adopted in this paper. It can not only reduce the calculation, also wholly reveal economy. What is more, this method has obtained comprehensive evaluation design result, achieving a better practical effect.

(3) When improving gear noise of existing automotive transmission, on the condition of not changing the values of pressure angle, modulus and spiral angle, it is feasible to use the optimization method due to reducing the meshing noise of two teeth meshing.

\section{REFERENCES}

[1] Z. Zhong, "Application and Design Discussion of 'Fine-pitch' Gear", QICHE CHILUN, pp. 1-7, February 2008.

[2] X. Zhengzhuang, Design on High Strength Gear, Beijing: China Machine Press, June 1981.

[3] F. Zhao, T. Wang, and H. Li, "Fuzzy optimization design method on fine-pitch tooth profile", Advanced Materials Research, vol. 156-157, pp. 1029-1036, 2011.

[4] Z. Jing, and W. Tie, "The fuzzy reliability optimum design of double circular arc gears transmission", SHANXI MACHINERY, pp. 14-16, February 2001.

[5] S. Zheng-Yu, D. Wei-Dong, and J. U. Yuan-Ping, "Optimum Design of Hypoid Gear with Noise Condition Considered", Journal of Wuhan Yejin University of Science and Technology, Vol. 28, pp. 52-55, January 2005.

[6] S. Hong-Sheng, "The Optimization Design of Planetary Gear Reducer", Equipment Manufacturing Technology, pp. 51-53, June 2009.

[7] L. Guoxiang, C. Conggui, L. Rong-Di, "Fuzzy reliability constraint hypoid gear design", Journal of Mechanical Transmission, pp. 4546, January 2001. 